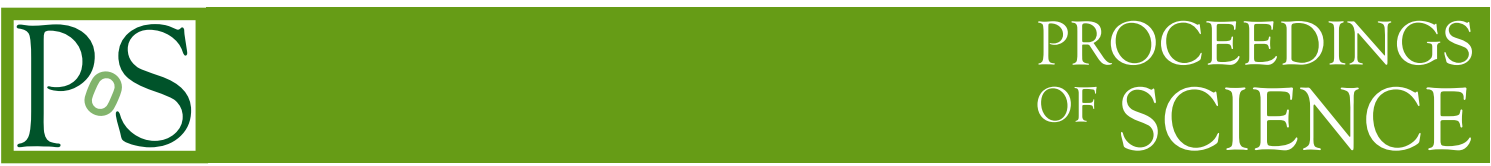

\title{
Combined Upper Limit for SM Higgs at the Tevatron
}

\author{
Björn Penning for the D $\varnothing$ and CDF Collaboration \\ Fermi National Accelerator Laboratory \\ E-mail: penning@fnal.gov
}

We combine results from CDF and $\mathrm{D} \emptyset$ on direct searches for a standard model (SM) Higgs boson $(H)$ in $p \bar{p}$ collisions at the Fermilab Tevatron at $\sqrt{s}=1.96 \mathrm{TeV}$. Compared to the previous Higgs Tevatron combination, more data and new channels $(W H \rightarrow \tau \nu b \bar{b}, V H \rightarrow \tau \tau b \bar{b} / j j \tau \tau$, $V H \rightarrow j j b \bar{b}, t \bar{t} H \rightarrow t \bar{t} b \bar{b}$ ) have been added. Most previously used channels have been reanalyzed to gain sensitivity. We use the latest parton distribution functions and $g g \rightarrow H$ theoretical cross sections when comparing our limits to the SM predictions. With 2.0-3.6 $\mathrm{fb}^{-1}$ of data analyzed at CDF, and 0.9-4.2 $\mathrm{fb}^{-1}$ at DØ, the $95 \%$ C.L. upper limits on Higgs boson production are a factor of 2.5 (0.86) times the SM cross section for a Higgs boson mass of $m_{H}=115(165) \mathrm{GeV} / \mathrm{c}^{2}$. Based on simulation, the corresponding median expected upper limits are 2.4 (1.1). The mass range excluded at $95 \%$ C.L. for a SM Higgs has been extended to $160<m_{H}<170 \mathrm{GeV} / \mathrm{c}^{2}$.

European Physical Society Europhysics Conference on High Energy Physics, EPS-HEP 2009,

July 16 - 222009

Krakow, Poland 


\section{Introduction}

The search for the Higgs boson is one of the main goals in the current High Energy Physics research. In this paper we report an udpdate of the combined searches performed by the CDF and $\mathrm{D} \emptyset$ collaborations, using the data collected at Tevatron collider at $\sqrt{s}=1.96 \mathrm{TeV}$. This update, covering the mass range $100-200 \mathrm{GeV} / \mathrm{c}^{2}$, includes not only an increased data set, but also the improvements in the analysis techniques. The various analysis are utilizing different production modes. The Higgs boson signal is produced in association with vector bosons $(q \bar{q} \rightarrow W / Z H)$, gluon-fusion $(g g \rightarrow H)$ and vector boson fusion $\left(q \bar{q} \rightarrow q^{\prime} \bar{q}^{\prime} H\right)$ corresponding to integrated luminosities from 2.0-3.6 $\mathrm{fb}^{-1}$ at CDF and 0.4-4.2 $\mathrm{fb}^{-1}$ at $\mathrm{D} \emptyset$. The Higgs boson decays analyzed are $H \rightarrow b \bar{b}, H \rightarrow W^{+} W^{-}, H \rightarrow \tau \tau$ and $H \rightarrow \gamma \gamma$. In total 75 mutually exclusive channels (23 for CDF and 52 for D0) are combined which are detailed in Ref. [1].

\section{Even Selection}

In this section we describe the most important selection requirements used by the analyses being combined. Event selection criteria are similar for the corresponding CDF and DØ analyses. In the case of $W H \rightarrow \ell v b \bar{b}$, an isolated lepton with one or more $b$-tagged jet, i.e. identified as containing a weakly-decaying $B$ hadron, is required. CDF considers as well events with an isolated track failing lepton identification requirements, thus providing some acceptance for single prong tau decays. The selected events must also display a significant imbalance in transverse momentum (referred to as missing transverse energy or $\mathbb{E}_{\mathrm{T}}$ ). The selection of the $Z H \rightarrow v v b \bar{b}$ analyses is similar, except events with isolated leptons are vetoed and stronger multijet background suppression techniques are applied. Both experiments use an additional track-based missing transverse momentum calculation as a discriminant against false $\mathbb{E}_{\mathrm{T}} . W H \rightarrow \ell v b \bar{b}$ events in which the lepton escapes detection will be selected by $Z H \rightarrow v v b \bar{b}$ searches. The search $Z H \rightarrow \ell \ell b \bar{b}$ is performed requiring two isolated leptons and at least two jets. Two and three jet events are analyzed separately and both collaboration define non-overlapping $b$-tagged samples with respect to the mis-identification rate of the $b$-tagging algorithm. The limits are set on final discriminants, typically neural network $(\mathrm{NN})$, boosted decision trees (BDT) and matrix element probabilities (ME) discriminants, but as well others like dijet mass and likelihoods are used.

For the $H \rightarrow W^{+} W^{-} \rightarrow \ell^{+} v \ell^{-} v$ analysis the events are characterized by large missing transverse energy $\mathbb{E}_{\mathrm{T}}$ and two isolated, oppositely charged leptons. D $\varnothing$ selects events using electrons or muons in the final state: $e^{+} e^{-}, e^{ \pm} \mu^{\mp}, \mu^{ \pm} \mu^{\mp}$. CDF separates events in five non-overlapping samples according to the purity of the lepton selection categories, and also split by the number of jets: 0,1 , or $2+$ jets. The sample with two or more jets is not split into signal purity categories. The final discriminants are neural-network outputs for $\mathrm{D} \emptyset$ and neural-network outputs including ME probabilities as input to the neural network, for CDF, in the 0-jet bin, else the ME are not used. Other analyses included in the combination are a search for Higgs bosons decaying to a tau lepton pairs, a new all-hadronic channel, $W H+Z H \rightarrow j j b \bar{b}, W H \rightarrow W W^{+} W^{-}$searches, a search for direct Higgs boson production decaying to a photon pair and search for the decay $t \bar{t} \rightarrow H$.

The data and predictions can be aggregated in bins of signal-to-background ratio, $s / b$. Data with similar $s / b$ may be added together with no loss in sensitivity, assuming similar systematic 

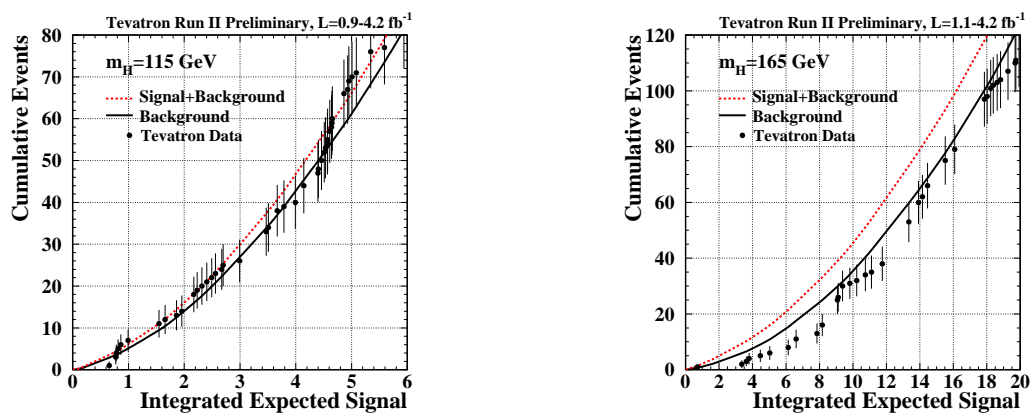

Figure 1: Integrated distributions of $s / b$, starting at the high $s / b$ side. The total signal+background and backgroundonly integrals are shown separately, along with the data sums. Data are only shown for bins that have data events in them.

errors. Integrating these distributions from the high- $s / b$ side downwards, we show the sums of signal, background, and data for the most pure portions of the selection of all channels added together. These integrals are displayed in Figure 1.

\section{Limit Calculations}

To gain confidence that the final result does not depend on the details of the statistical formulation, we perform two types of combinations, using the Bayesian and Modied Frequentist approaches, which give similar results (within 10\%). Both methods rely on distributions in the final discriminants and not just on their single integrated values. Systematic uncertainties enter as uncertainties on the expected number of signal and background events, as well as on the distribution of the discriminants in each analysis ("shape uncertainties"). Both methods use likelihood calculations based on Poisson probabilities.

\subsection{Systematic Uncertainties}

Systematic uncertainties differ between experiments and analyses, and they affect the rates and shapes of the predicted signal and background in correlated ways. The combined results incorporate the sensitivity of predictions to values of nuisance parameters, and include correlations, between rates and shapes, between signals and backgrounds, and between channels within experiments and between experiments. We correlate various systematics like the uncertainties on the integrated luminosity and production cross sections. Other systematics like for example the $b$-tagging uncertainty or $Q C D$ multijet background cannot be correlated due to the different techniques employed, but these systematics are correlated within each experiment. All systematic uncertainties arising from the same source are taken to be correlated between the different backgrounds and between signal and background.

\section{Combined Results}

Using the combination procedures outlined in Section III, we extract limits on SM Higgs boson production $\sigma \times B(H \rightarrow X)$ in $p \bar{p}$ collisions at $\sqrt{s}=1.96 \mathrm{TeV}$ for $m_{H}=100-200 \mathrm{GeV} / \mathrm{c}^{2}$. 
To facilitate comparisons with the standard model and to accommodate analyses with different degrees of sensitivity, we present our results in terms of the ratio of obtained limits to cross section in the SM, as a function of Higgs boson mass. A value of the combined limit ratio which is less than or equal to one would indicate that that particular Higgs boson mass is excluded at the $95 \%$ C.L.

The combinations of results of each single experiment, as used in this Tevatron combination, yield the following ratios of 95\% C.L. observed (expected) limits to the SM cross section: 3.6 (3.2) for $\mathrm{CDF}$ and 3.7 (3.9) for $\mathrm{D} \emptyset$ at $m_{H}=115 \mathrm{GeV} / \mathrm{c}^{2}$, and 1.5 (1.6) for $\mathrm{CDF}$ and 1.3 (1.8) for $\mathrm{D} \emptyset$ at $m_{H}=165 \mathrm{GeV} / \mathrm{c}^{2}$.

The ratios of the $95 \%$ C.L. expected and observed limit to the SM cross section are shown in Fig. 2 for the combined CDF and DØ analyses. The observed and median expected ratios are listed for the tested Higgs boson masses in Table 1 for $m_{H} \leq 150 \mathrm{GeV} / \mathrm{c}^{2}$, and in Table 2 for $m_{H} \geq 155 \mathrm{GeV} / \mathrm{c}^{2}$, as obtained by the Bayesian and the $C L_{S}$ methods. In the following summary we quote only the limits obtained with the Bayesian method since they are slightly more conservative (based on the expected limits) for the quoted values, but all the equivalent numbers for the $C L_{S}$ method can be retrieved from Ref. [1] We obtain the observed (expected) values of 2.5 (2.4) at $m_{H}=115 \mathrm{GeV} / \mathrm{c}^{2}, 0.99(1.1)$ at $m_{H}=160 \mathrm{GeV} / \mathrm{c}^{2}, 0.86(1.1)$ at $m_{H}=165 \mathrm{GeV} / \mathrm{c}^{2}$, and 0.99 (1.4) at $m_{H}=170 \mathrm{GeV} / \mathrm{c}^{2}$. We exclude at the $95 \%$ C.L. the production of a standard model Higgs boson with mass between 160 and $170 \mathrm{GeV} / \mathrm{c}^{2}$. This result is obtained with both Bayesian and $C L_{S}$ calculations.

\begin{tabular}{lccccccccccc}
\hline & 100 & 105 & 110 & 115 & 120 & 125 & 130 & 135 & 140 & 145 & 150 \\
\hline Expected & 2.0 & 2.0 & 2.2 & 2.4 & 2.7 & 2.9 & 2.9 & 2.7 & 2.5 & 2.4 & 1.8 \\
Observed & 1.9 & 1.8 & 2.4 & 2.5 & 2.8 & 3.0 & 3.5 & 2.4 & 2.7 & 2.8 & 1.9 \\
\hline
\end{tabular}

Table 1: Ratios of median expected and observed 95\% C.L. limit to the SM cross section for the combined CDF and $\mathrm{D} \emptyset$ analyses as a function of the Higgs boson mass in $\mathrm{GeV} / \mathrm{c}^{2}$, obtained with the Bayesian and with the $C L_{S}$ method.

\begin{tabular}{lcccccccccc}
\hline & 155 & 160 & 165 & 170 & 175 & 180 & 185 & 190 & 195 & 200 \\
\hline Expected & 1.5 & 1.1 & 1.1 & 1.4 & 1.6 & 1.9 & 2.2 & 2.7 & 3.5 & 4.2 \\
Observed & 1.4 & 0.99 & 0.86 & 0.99 & 1.1 & 1.2 & 1.7 & 2.0 & 2.6 & 3.3 \\
\hline
\end{tabular}

Table 2: Ratios of median expected and observed 95\% C.L. limit to the SM cross section for the combined CDF and $\mathrm{D} \emptyset$ analyses as a function of the Higgs boson mass in $\mathrm{GeV} / \mathrm{c}^{2}$, obtained with the Bayesian and with the $C L_{S}$ method.

In summary, we have combined all available CDF and DØ results on SM Higgs search, based on luminosities ranging from 0.9 to $4.2 \mathrm{fb}^{-1}$. Compared to our previous combination, new channels have been added and most previously used channels have been reanalyzed to gain sensitivity. We use the latest parton distribution functions and $g g \rightarrow H$ theoretical cross sections when comparing our limits to the SM predictions at high mass. The 95\% C.L. upper limits on Higgs boson production are a factor of $2.5(0.86)$ times the SM cross section for a Higgs boson mass of $m_{H}=115(165) \mathrm{GeV} / \mathrm{c}^{2}$. The corresponding median expected upper limits are 2.4 (1.1). These results extend significantly the individual limits of each collaboration and our previous combination. The mass range excluded at $95 \%$ C.L. for a SM Higgs has been extended to 


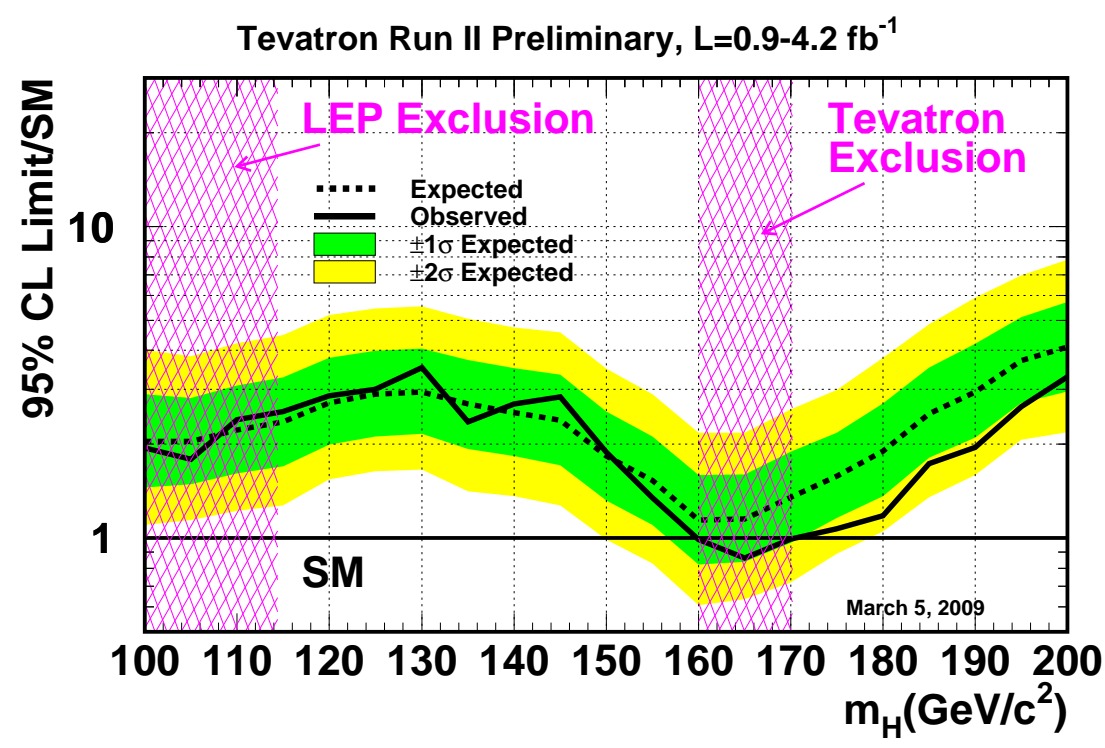

Figure 2: Observed and expected (median, for the background-only hypothesis) 95\% C.L. upper limits on the ratios to the SM cross section, as functions of the Higgs boson mass for the combined CDF and D $\varnothing$ analyses. The limits are expressed as a multiple of the SM prediction for test masses (every $5 \mathrm{GeV} / \mathrm{c}^{2}$ ) for which both experiments have performed dedicated searches in different channels. The points are joined by straight lines for better readability. The bands indicate the $68 \%$ and $95 \%$ probability regions where the limits can fluctuate, in the absence of signal. The limits displayed in this figure are obtained with the Bayesian calculation.

$160<m_{H}<170 \mathrm{GeV} / \mathrm{c}^{2}$. The sensitivity of our combined search is expected to grow substantially in the near future with the additional luminosity already recorded at the Tevatron and not yet analyzed, and with additional improvements of our analysis techniques which will be propagated in the current and future analyses.

\section{References}

[1] DØ Collaboration, "Combined upper limits on standard model Higgs boson production from the DØ experiment with up to $4.2 \mathrm{fb}^{-1}$ of data" DØ Conference Note 5896 (2009).

[2] CDF Collaboration, "Combined Upper Limit on Standard Model Higgs Boson Production for Winter 2009”, CDF Conference Note 9674 (2009).

[3] DØ Collaboration, "Search for the Standard Model Higgs boson in $\gamma \gamma$ final state with $4.2 \mathrm{fb}^{-1}$ data", DØ Conference Note 5858.

[4] T. Junk, Nucl. Instrum. Meth. A434, p. 435-443, 1999, A.L. Read, "Modified frequentist analysis of search results (the $C L_{s}$ method)", in F. James, L. Lyons and Y. Perrin (eds.), Workshop on Confidence Limits, CERN, Yellow Report 2000-005, available through cdsweb. cern.ch.

[5] W. Fisher, "Systematics and Limit Calculations," FERMILAB-TM-2386-E. 